\title{
The Influence of Temperature on C153 Steady-State Absorption and Fluorescence Kinetics in Hydrogen Bonding Solvents
}

\author{
Krzysztof Dobek • Jerzy Karolczak
}

Received: 4 April 2012 / Accepted: 30 July 2012

(C) The Author(s) 2012. This article is published with open access at Springerlink.com

\begin{abstract}
In a recent paper (J Fluoresc (2011) 21:15471557) a temperature induced modulation of Coumarin 153 (C153) fluorescence lifetime and quantum yield for the probe dissolved in the polar, nonspecifically interacting 1chloropropane was reported. This modulation was also observed in temperature dependencies of the radiative and nonradiative rates. Here, we show that the modulation is also observed in another 1-chloroalkane-1-chlorohexane, as well as in hydrogen bonding propionitrile, ethanol and trifluoroethanol. Change in the equilibrium distance between $S_{0}$ an $S_{1}$ potential energies surfaces was identified as the source of this modulation. This change is driven by temperature changes. It leads to a modulation of the fluorescence transition dipole moment and it is the primary source of the experimental effects observed. Additionally, we have found that proticity of the solvent induces a rise in the fluorescence transition dipole moment, which leads to a shortening of the fluorescence lifetime. Hydrogen bonds are formed by $\mathrm{C} 153$ also with hydrogen accepting solvents like propionitrile. We show that while such bonds do not affect the transition probability, they do change the $S_{0}$ an $S_{1}$ energy gap which in turn implies a change in non-radiative transition rate in a similar way as in protic solvents, as well as in the fluorescence spectrum position. Finally, the influence of temperature on the energies of hydrogen bonds formed by $\mathrm{C} 153$ when acting as hydrogen donor or acceptor is reported.
\end{abstract}

\footnotetext{
K. Dobek $(\bowtie) \cdot J$. Karolczak

Faculty of Physics, Adam Mickiewicz University, Umultowska 85,

61-614 Poznań, Poland

e-mail: dobas@amu.edu.pl

J. Karolczak

Center For Ultrafast Laser Spectroscopy,

Adam Mickiewicz University,

Umultowska 85,

61-614 Poznań, Poland
}

Keywords Coumarin 153 - Thermochromism · Solvation · Temperature $\cdot$ Transition dipole moment $\cdot$ Hydrogen bond

\section{Introduction}

The thermochromism of simple dye molecules is a good indicator of changes in the dye environment polarity following changes in temperature. In many cases thermochromic shifts in absorption and emission reflect the temperature induced changes in refractive index, $n$, and electric permittivity, $\varepsilon$, of the solvent as observed by our group in $[1,2]$ for 4-aminophthalimide (4-AP) and Coumarin 153 (C153, Scheme 1) dissolved in several polar non-protic 1-chloroalkanes, or by Suppan et al. in [3-6] for several probes including 4-AP.

However, for a dye interacting specifically with the solvent molecules, temperature influence on the energy of this interaction can affect much more significantly the scale of thermochromic shifts than for a dye showing nonspecific interaction, related to $n(\mathrm{~T})$ and $\varepsilon(\mathrm{T})$ dependencies. This additional shift can be of the sign the same as or the opposite to that resulting from nonspecific interactions. In $[1,2]$ we have found both 4-AP and C153 to form hydrogen bonds (H-bond) acting as hydrogen acceptors and donors. Both types of H-bonding interactions result in an additional stabilisation of the first excited singlet state $S_{1}$ of both probes. However, in the case of the probes acting as hydrogen acceptors, this additional stabilization has been found to weaken with decreasing temperature. In the case of the dyes acting as hydrogen donors, a slightly rise in the stabilization was observed with decreasing temperature. To identify the origin of such temperature changes in H-bond energies, a preliminary study on the influence of temperature on absorption and emission, including fluorescence time-resolved measurements, was performed for $\mathrm{C} 153$ dissolved in 1chloropropane (ClP) [7]. The purpose of this study was to 


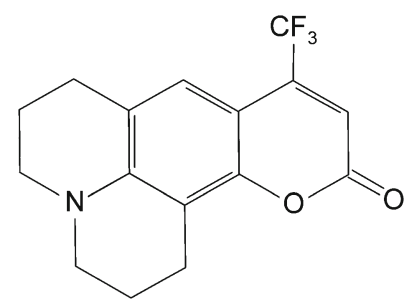

Scheme 1 Coumarin 153 (C153) structure

determine the influence of temperature on the kinetics of C153 deactivation from $\mathrm{S}_{1}$ in nonspecifically interacting polar solvents. Surprisingly, C153 fluorescence lifetime $\left(\tau_{\mathrm{F}}\right)$ and fluorescence quantum yield $\left(\phi_{\mathrm{F}}\right)$ have been found to follow in this solvent a complex temperature dependence, indicating the intramolecular deactivation is not only controlled by the energy gap law of radiationless deactivation rate.

In this report we would like to present results of our further studies of temperature influence on $\mathrm{C} 153$ excitation and deactivation. In order to compare new results with the previous ones obtained for ClP [7], the absorption and emission spectra as well as quantum yield and fluorescence lifetime were measured for $\mathrm{C} 153$ dissolved in 1-chlorohexane $(\mathrm{ClH})$ in the temperature range of $183 \mathrm{~K}-323 \mathrm{~K}$. Additionally, the same spectra and quantities were determined in propionitrile $(\mathrm{PPN})$ - a hydrogen acceptor (Kamlet-Taft polarity scale $\alpha=0, \beta=0.4$ [8-10]), in trifluoroethanol (TFEtOH) - a hydrogen donor $(\alpha=1.51, \beta=0)$ and in ethanol $(\mathrm{EtOH})$ - a hydrogen donor and acceptor $(\alpha=0.86, \beta=0.75)$. The choice of these solvents was dictated by their H-bonding character and by their melting points, the lower the better.

\section{Methods}

As previously, emission spectra were accumulated using a modified Aminco SPF-500 spectrofluorimeter with single photon counting detection. Absorption spectra were measured using a Jasco V-550 spectrometer. Temperature control was performed using an Oxford Instruments Optistat DN cryostat. Time-resolved fluorescence measurements were made using a TCSPC system with an instrument response function (IRF) of $30 \mathrm{ps}$ full-width at half of the maximum (FWHM). The time per channel was set to $12.2 \mathrm{ps}$ and fluorescence decays were collected into 4,096 channels [11]. A home-made analytical software was used to fit the decays with the simplex approximation algorithm. The signal scattered at excitation light wavelength from a ludox in water solution was used as the IRF. C153 (Fluka) was used as received, 1-chlorohexane (Aldrich), propionitrile (Sigma-Aldrich), ethanol (Sigma-Aldrich) and trifluoroethanol (Sigma-Aldrich) were dehydrated using $3 \AA$
(Merck) and $4 \AA$ (Fluka) molecular sieves and the sample were prepared under argon atmosphere after solvent dehydration. C153 concentration was kept at $\sim 10^{-5} \mathrm{M}$. It is worth underlining the necessity of careful dehydration, whose failure can lead to incorrect results as shown for 4-AP in [12]. In this report we also show results obtained for $\mathrm{C} 153$ dissolved in $\mathrm{ClH}$ contaminated with water.

\section{Results}

\section{Steady-State Results}

Steady-state absorption and emission spectra were measured in selected solvents at different temperature ranges with a $10 \mathrm{~K}$ step. Figure 1 present normalized absorption spectra of $\mathrm{C} 153$ in all solvents used, obtained at room temperature and at $233 \mathrm{~K}$, for the sake of comparison also the ones obtained in 1-chloropropane [7] are shown.

A subtle difference in the shape of the spectra recorded at the two temperatures can be noted for ClP and $\mathrm{ClH}$. To better resolve this change it is convenient to determine the differences in absorbance at consecutive temperatures at which measurements were made, separated by $10 \mathrm{~K}$. Figure 2 presents such "derivatives" for $\mathrm{C} 153$ in $\mathrm{ClH}$.

Note that the curves shown in Fig. 2 include the effects resulting from solvatochromic shifts of absorption, thus they cannot be directly interpreted as a result of temperature induced intramolecular changes. Nevertheless, in $\mathrm{ClH}$ (and ClP, not shown) a vibronic structure of the changes in absorbance can be seen. In Fig. 2 the distance in energy of $1,380 \mathrm{~cm}^{-1}$ is drawn separating two consecutive maxima of the curve for the lowest temperature change. This value is close to the $1,150 \mathrm{~cm}^{-1}$ frequency of the deactivation accepting mode found in the emission in ClP [7] and in gas-phase $[13,14]$. In other solvents no structure can be observed which would well correspond to the lack of any clear change in absorption spectra shape when decreasing temperature. Note that the ratio of the amplitude of two maxima visible in each curve in Fig. 2 changes with decreasing $\mathrm{T}$ in favour of the highest most red shifted peak.

Figure 3 shows the temperature dependence of the peak positions, $\nu_{\mathrm{p}}$, of absorption spectrum $\mathrm{S}_{0} \rightarrow \mathrm{S}_{1}$ band in all five solvents. Lines represent $\nu_{\mathrm{p}}(\mathrm{T})$ slopes predicted theoretically in the way described later in the text.

In PPN, EtOH and TFEtOH the peak positions correspond to $\nu_{\mathrm{p}}$ of LogNormal curves fitted to the absorption spectra. For $\mathrm{ClP}$ and $\mathrm{ClH}$, due to the vibronic structure of the spectra, no correct fit could be obtained by means of LogNormal functions. Thus, in this case $\nu_{\mathrm{p}}$ corresponds to frequencies at which the maxima of the consecutive absorption spectra were observed. The difference in approaches 
Fig. 1 C153 absorption spectra at room temperature (a) and at $233 \mathrm{~K}$ (b) in: $\mathrm{ClP}$ (solid), $\mathrm{ClH}$ (long dash), PPN (short dash), $\mathrm{EtOH}($ dot-dash) and TFEtOH (dot)
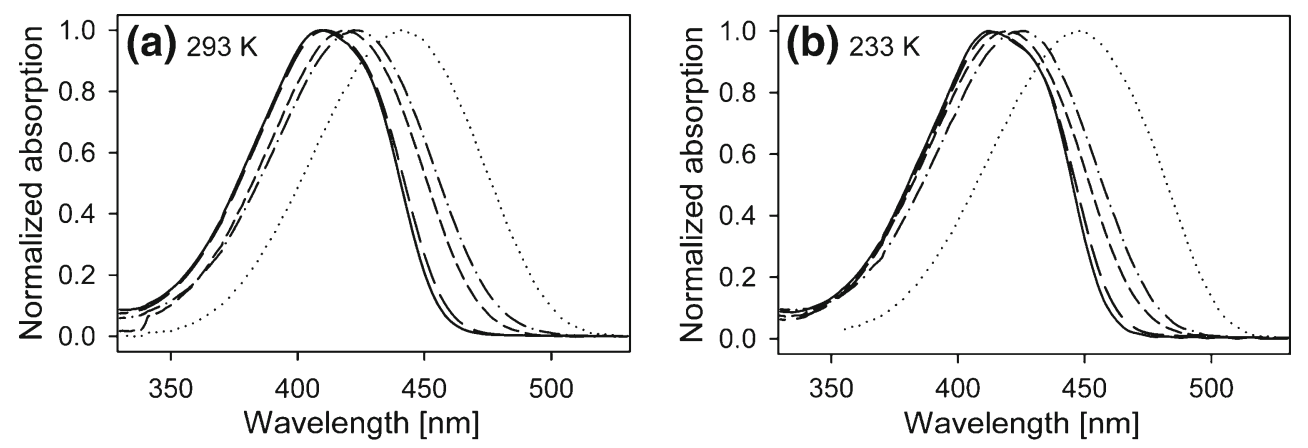

results in a smoother $\mathrm{T}$ dependence in the three specifically interacting solvents.

Using quinine sulphate in $0.05 \mathrm{M} \mathrm{H}_{2} \mathrm{SO}_{4}\left(\phi_{\mathrm{F}}=0.52\right)$ as a standard, C153 fluorescence quantum yield, $\phi_{\mathrm{F}}$, in all solvents was determined at room temperature. Then, by assuming the room temperature emission spectrum in selected solvent as a standard, temperature dependencies of $\phi_{\mathrm{F}}$ were determined, shown in Fig. 4.

Values of $\phi_{\mathrm{F}}(\mathrm{T})$ follow similar dependencies in $\mathrm{ClH}$ and PPN, while in EtOH and TFEtOH they are significantly different. Note, that $\phi_{\mathrm{F}}(\mathrm{T})$ in TFEtOH is quite similar to the one found in ClP [7]. Only in EtOH $\phi_{\mathrm{F}}$ rises at the lowest temperatures. Analysis of time-resolved emission spectra (TRES), discussed in the next section, have shown that this rise is a consequence of a significant retardation of solvation dynamics in $\mathrm{EtOH}$ at low temperatures. In all solvents $\phi_{\mathrm{F}}(\mathrm{T})$ slope changes from negative into positive at distinct temperatures: $243 \mathrm{~K}$ in $\mathrm{ClH}, 273 \mathrm{~K}$ in PPN, $273 \mathrm{~K}$ in EtOH and $283 \mathrm{~K}$ in TFEtOH. In ClP it was $273 \mathrm{~K}$. The changes observed in $\phi_{\mathrm{F}}$ are twice as high in $\mathrm{ClH}$ and PPN as those in EtOH and TFEtOH.

\section{Time-Resolved Results}

First of all, fluorescence decays were measured for C153 in all solvents in the same temperature ranges as in the case of

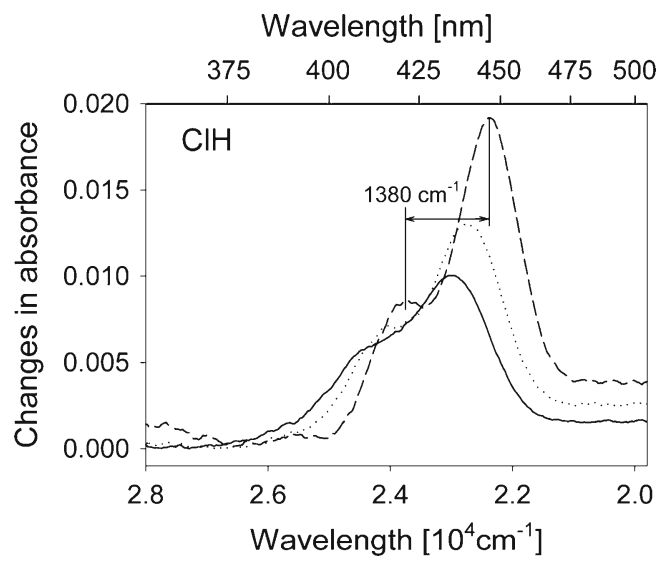

Fig. 2 Changes in absorbance of $\mathrm{C} 153$ in $\mathrm{ClH}$ induced by decrease in temperature: $323 \mathrm{~K} \rightarrow 313 \mathrm{~K}$ (solid line), $263 \mathrm{~K} \rightarrow 253 \mathrm{~K}($ dot $)$ and $193 \mathrm{~K} \rightarrow 183 \mathrm{~K}$ (dash) steady-state absorption and emission spectra. Due to some limitation of our experimental setup, during the experiment the excitation wavelength was constant and set to the maximum of the room temperature absorption spectrum in a selected solvent, while the emission wavelength was set to the maximum of the emission spectrum at a selected temperature. In $\mathrm{ClH}$ and PPN the decay was found to be properly described by a single exponential decay function in the full temperature range. In EtOH below $293 \mathrm{~K}$ and in TFEtOH in the full $\mathrm{T}$ range a double exponential fit was necessary to correctly reconstruct the data. The double exponential function consisted of a dominant long component. A decrease in temperature induced a change in the second component decay time from $100 \mathrm{ps}$ (contribution $\mathrm{F}=0.2 \%$ ) to $2,200 \mathrm{ps}(\mathrm{F}=25 \%)$ in $\mathrm{EtOH}$, and from $100 \mathrm{ps}(\mathrm{F}=0.6 \%)$ to $900 \mathrm{ps}(\mathrm{F}=4 \%)$ in TFEtOH. The long component decay time had been found in both protic solvents to follow a similar temperature dependence as the single exponential decay time, also fitted to the data. Therefore, Fig. 5 shows $\tau_{\mathrm{F}}$ of the single exponential fit for $\mathrm{ClH}$, PPN and the long component of the double exponential fit for both protic solvents.

There is a similarity in $\tau_{\mathrm{F}}(\mathrm{T})$ dependencies in $\mathrm{ClH}$ and PPN, as well as in EtOH and TFEtOH. Similarly, as for $\phi_{\mathrm{F}}(\mathrm{T})$ there are characteristic temperatures at which changes in $\tau_{\mathrm{F}}(\mathrm{T})$ slope are observed in $\mathrm{ClH}(263 \mathrm{~K})$ and $\mathrm{PPN}$

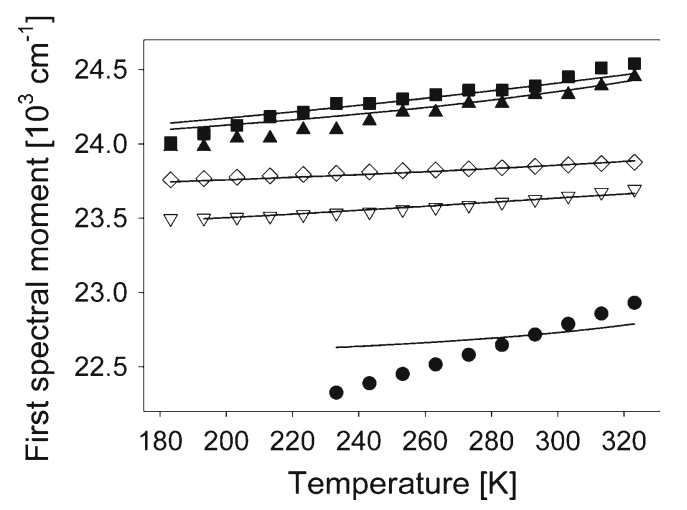

Fig. $3 \mathrm{C} 153$ steady-state absorption spectra maximum positions $\left(\nu_{\mathrm{p}}\right)$ vs temperature in: $\mathrm{ClH}$ (filled squares), $\mathrm{ClP}$ (filled triangles), PPN (empty diamonds), EtOH (empty triangles) and TFEtOH (filled circles). Lines correspond to theoretically determined positions 
Fig. 4 Temperature dependencies of the fluorescence quantum yield, $\phi_{\mathrm{F}}$, of $\mathrm{C} 153$ in $\mathrm{ClH}(\mathbf{a}), \mathrm{PPN}(\mathbf{b}), \mathrm{EtOH}(\mathbf{c})$ and TFEtOH (d)

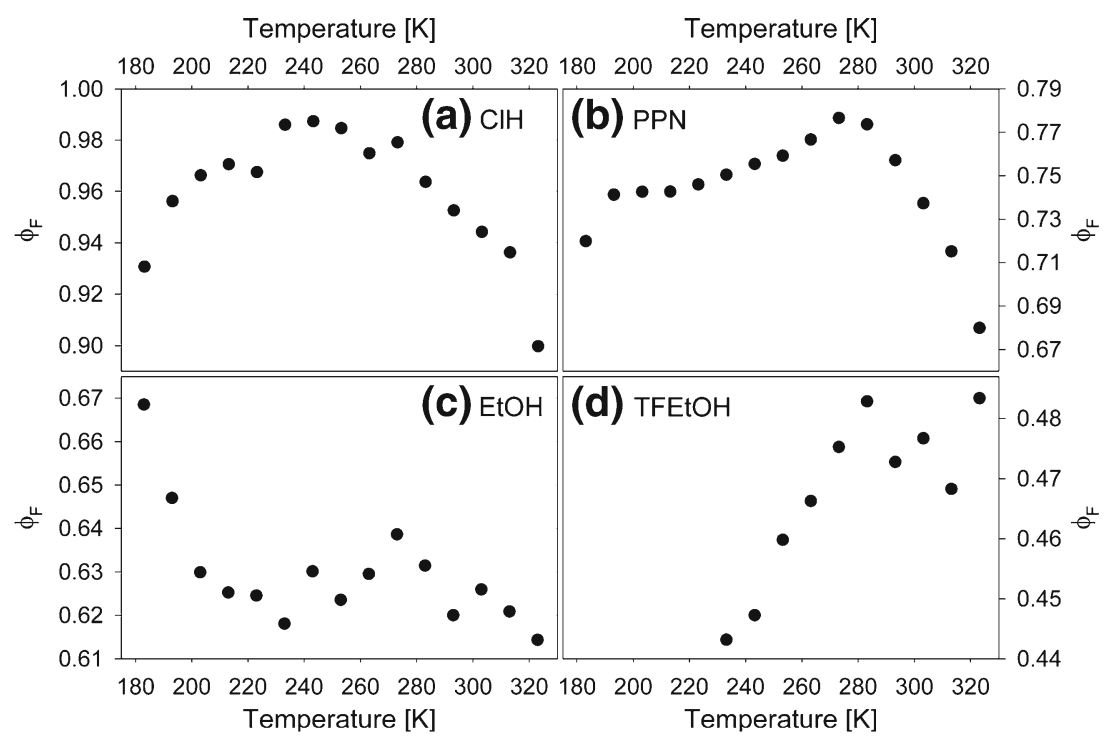

(273 K). In alcohols a modulation of $\tau_{\mathrm{F}}(\mathrm{T})$ can be observed in $\mathrm{T}$ ranges between $300 \mathrm{~K}$ and $273 \mathrm{~K}$, the same in which changes in $\phi_{\mathrm{F}}(\mathrm{T})$ slope occur. The scale of the changes in $\tau_{\mathrm{F}}$ seems to be linked to the polarity of the solvent, the higher the polarity the larger the range of the $\tau_{\mathrm{F}}$ values. This observation includes the results for C153 in ClP from [7].

Effects of improper dehydration of 1-chloroalkanes on 4-AP thermochromism were reported in Ref. [12]. For $\mathrm{C} 153$ in $\mathrm{ClP}$ and $\mathrm{ClH}$ we also noticed that incorrect dehydration of these solvents led to the $\tau_{\mathrm{F}}(\mathrm{T})$ dependencies significantly different from that shown in Fig. 5 and in Ref. [7]. A similar observation was also made for $\mathrm{C} 153$ in PPN. Thus, following the procedure undertaken in Ref. [12] we had preliminarily dehydrated $50 \mathrm{~mL}$ of $\mathrm{ClH}$ and next we added to it $2 \mu \mathrm{L}$ of distilled water, which corresponded to a $2.2 \cdot 10^{-3} \mathrm{M}$ water concentration.
Fluorescence decays in such a $\mathrm{ClH}+$ water mixture were measured at the wavelengths corresponding to the maxima of steady-state spectra collected at subsequent temperatures. They were found to be described by double exponential decay functions with a dominant 5-6 ns component and a minor $\sim 200$ ps second component whose contribution did not exceed $1 \%$. Figure $6 \mathrm{a}$ presents the temperature dependence of the long component for $\mathrm{C} 153$ dissolved in $\mathrm{ClH}+$ water mixture along with the dependence found in dehydrated $\mathrm{ClH}$ shown already in Fig. 5. Additionally, Fig. 6b shows the impact of improper dehydration of PPN on $\mathrm{C} 153 \tau_{\mathrm{F}}(\mathrm{T})$ dependence. The properly dehydrated sample (filled circles) was prepared with PPN dried with molecular sieves twice as long as for the improperly dehydrated one (empty circles), after changing the molecular sieves once. It was also
Fig. 5 Temperature dependencies of the fluorescence decay time for $\mathrm{C} 153$ in $\mathrm{ClH}$ (a), PPN (b), EtOH (c) and TFEtOH (d). In the alcohols the dominant long component time (filled circles) of the doubleexponential decay function fitted to the experimental decays is presented along with the single exponential decay time for TFEtOH (empty circles)

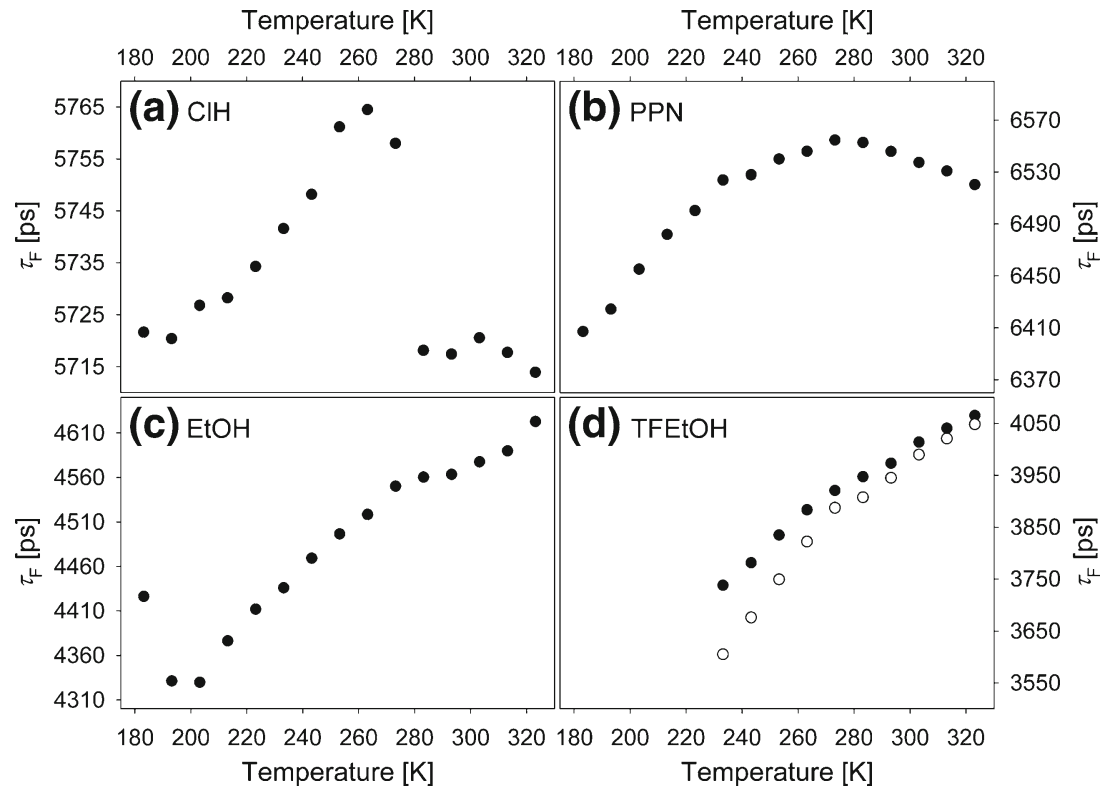


Fig. 6 Temperature

dependence of fluorescence

decay time of $\mathrm{C} 153$ in

dehydrated $\mathrm{ClH}$ (a)-filled

circles, in $\mathrm{ClH}+$ water mixture at

$2.2 \cdot 10^{-3} \mathrm{M}$ water concentration

(a)-empty circles, in dehydrated

PPN (b)-filled circles and in improperly dehydrated PPN

(b)-empty circles

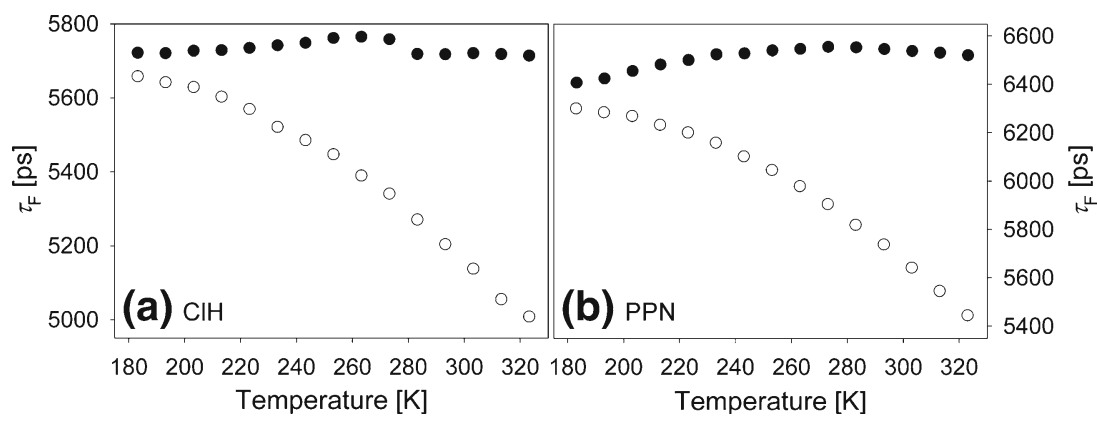

the two other solvents. Such a conclusion follows from analysis of TRES determined for $\mathrm{C} 153$ in EtOH at $293 \mathrm{~K}$, $233 \mathrm{~K}$ and $193 \mathrm{~K}$ and in TFEtOH at $293 \mathrm{~K}$ and $233 \mathrm{~K}$. TRES were determined in a usual way. First, fluorescence decays at 12 selected wavelengths with a $15 \mathrm{~nm}$ step were determined in EtOH, starting from $480 \mathrm{~nm}$, and in TFEtOH starting from $490 \mathrm{~nm}$. The experimental signals were fitted by multi-exponential decay functions. Next, the decay functions resulting from the fit were normalized in such a way as to equalize the area under the decay to the steady-state fluorescence spectrum intensity at selected wavelength and temperature. Steady-state spectra were corrected for the detector sensitivity and scaled by the $\lambda^{2}$ factor. From the normalized decays TRES were reconstructed. Next, each spectrum of the TRES corresponding to subsequent time delay was fitted by a LogNormal function, with the amplitude, asymmetry, peak frequency $\nu_{\mathrm{p}}$ and half-width as the parameters of the fit. From $\nu_{\mathrm{p}}$ values the correlation function $C(t)=\left(v_{p}(t)-v_{p}(\infty)\right) /\left(v_{p}(0)-v_{p}(\infty)\right)$ was determined. Here, $\nu_{\mathrm{p}}(\infty)$ corresponds to the peak frequency of the LogNormal curve fitted to the TRES spectrum at the longest time delay. Finally, $\mathrm{C}(\mathrm{t})$ dependencies were fitted by single- and double-exponential decays, depending on which

Table 1 Times and amplitudes of fluorescence decay components found for $\mathrm{C} 153$ in $\mathrm{ClH}+$ water mixture at $2.2 \times 10^{-3} \mathrm{M}$ water concentration and at selected temperatures and wavelengths

\begin{tabular}{llllll}
\hline $\mathrm{T}[\mathrm{K}]$ & $\lambda[\mathrm{nm}]$ & $\tau_{1}[\mathrm{ps}]$ & $\mathrm{a}_{1}$ & $\tau_{2}[\mathrm{ps}]$ & $\mathrm{a}_{2}$ \\
\hline 323 & 445 & 4494 & 0.82 & 130 & 0.18 \\
& 483 & 5008 & 0.93 & 190 & 0.07 \\
& 556 & 5011 & 0.98 & 281 & 0.02 \\
293 & 450 & 5152 & 0.75 & 87 & 0.25 \\
& 492 & 5204 & 0.94 & 207 & 0.06 \\
& 560 & 5221 & 1.07 & 16 & -0.07 \\
263 & 450 & 5397 & 0.88 & 2045 & 0.12 \\
& 490 & 5390 & 0.90 & 131 & 0.10 \\
& 563 & 5462 & 0.98 & 170 & 0.02 \\
213 & 445 & 5466 & 0.36 & 131 & 0.64 \\
& 490 & 5603 & 0.90 & 217 & 0.10 \\
& 560 & 5609 & 1.38 & 50 & -0.38 \\
\hline
\end{tabular}


number of exponential components was found necessary to reproduce $\mathrm{C}(\mathrm{t})$ properly. Table 2 presents the obtained $\mathrm{C}(\mathrm{t})$ decay time values, $\tau_{\mathrm{si}}$, along with the component amplitudes, $b_{i}$, and the average decay time defined as

$\left\langle\tau_{s}\right\rangle=\left(\sum_{i} b_{i} \tau_{s i}\right) / \sum_{i} b_{i}$

The values reported in Table 2 describe only the slowest part of $\mathrm{C}(\mathrm{t})$ decays, because of a small time-resolution used here. There is a good agreement between the value obtained for $\mathrm{C} 153$ in $\mathrm{EtOH}$ at $293 \mathrm{~K}$ and the longest $\mathrm{C}(\mathrm{t})$ component reported in [15]. The solvation dynamics is slower in TFEtOH at room $T$. However, if we analyze the relative change in $\tau_{\mathrm{s}}$ in EtOH and TFEtOH with decreasing T, we see that the solvation is slowed by decreasing T much more efficiently in EtOH. Additionally, this retardation grows quickly at the lowest temperatures in this solvent. This is the reason why $\phi_{\mathrm{F}}$ in Fig. 4 and $\tau_{\text {F }}$ in Fig. 5 increase in EtOH at the lowest T. Strongly retarded solvation at this $\mathrm{T}$ range prevents the steady-state spectrum from shifting fully to the red. As a result, fluorescence deactivation rate $\left(k_{F}\right)$ proportional to $\nu^{3}$ as well as to $\phi_{\mathrm{F}}$, increases. Additionally, $\phi_{\mathrm{F}}$ which is calculated by integration of the fluorescence steady-state spectrum, rises because the integration range is shifted to the blue, thus to higher energies. Finally, as mentioned earlier, the retarded solvation is responsible for the bi-exponential character of the fluorescence decays in the alcohols measured at the maximum of the steady-state fluorescence spectra. We assume in the next section that the dominant longest component of the fluorescence decays in EtOH and TFEtOH bring reliable information about $\mathrm{C} 153$ deactivation rate, at least in the range of high temperatures. However, for the lowest $\mathrm{T}$ such assumption is clearly invalid, thus the interpretation of the kinetic results presented below is correct for $\mathrm{C} 153$ in alcohols only in the high temperature range.

\section{Discussion}

Thermochromic shifts of $\mathrm{C} 153$ emission spectra reported in [2] indicated that the probe forms H-bond with hydrogen

Table 2 Decay time values, $\tau_{\mathrm{si}}$, and amplitudes, $\mathrm{b}_{\mathrm{i}}$, of the exponential decay components fitted to the solvation correlation function, $\mathrm{C}(\mathrm{t})$, determined for $\mathrm{C} 153$ in $\mathrm{EtOH}$ and $\mathrm{TFEtOH}$ at selected temperatures. $\left\langle\tau_{\mathrm{s}}\right\rangle$ - average decay time

\begin{tabular}{lllllll}
\hline Solvent & $\mathrm{T}[\mathrm{K}]$ & $\tau_{\mathrm{s} 1}[\mathrm{ps}]$ & $\mathrm{b}_{1}$ & $\tau_{\mathrm{s} 2}[\mathrm{ps}]$ & $\mathrm{b}_{2}$ & $\left\langle\tau_{\mathrm{s}}\right\rangle[\mathrm{ps}]$ \\
\hline EtOH & 293 & 35 & 1 & - & - & 35 \\
& 233 & 70 & 0.45 & 228 & 0.55 & 157 \\
& 193 & 208 & 0.35 & 974 & 0.65 & 704 \\
TFEtOH & 293 & 77 & 0.66 & 257 & 0.34 & 138 \\
& 233 & 50 & 0.23 & 378 & 0.77 & 302 \\
\hline
\end{tabular}

accepting solvents, and that the change in energy of this bond, stimulated by probe excitation, increases with decreasing temperature, at least in the vibrationally and solvent relaxed first singlet excited state, $S_{1}^{\text {rel }}$. These results were obtained as a difference between the emission spectrum positions predicted by the Onsager model and the experimental position values. To evaluate in a similar way the possible influence of specific interactions on absorption peak positions the Onsager model was applied using Eq. 1 $[2,16]$.

$$
\begin{aligned}
\Delta v_{A b s} & =2 \frac{\mu_{g}\left(\mu_{g}-\mu_{e}\right)}{a^{3}} \cdot\left(\frac{\varepsilon-1}{\varepsilon+2}-\frac{n^{2}-1}{n^{2}+2}\right) \\
& +\frac{\mu_{g}^{2}-\mu_{e}^{2}}{a^{3}} \cdot\left(\frac{n^{2}-1}{2 n^{2}+2}\right) \\
& +\frac{6 \mu_{g}^{2}\left(a_{g}-a_{e}\right)}{a^{6}} \cdot\left(\frac{\varepsilon-1}{\varepsilon+2}-\frac{n^{2}-1}{n^{2}+2}\right)^{2} \\
& +\frac{3\left(a_{g}-a_{e}\right) J J_{S}}{2 h c a^{3}\left(J+J_{S}\right)} \cdot \frac{n^{2}-1}{n^{2}+2}
\end{aligned}
$$

Above, $\mu_{\mathrm{g}}$ and $\mu_{\mathrm{e}}$ are $\mathrm{C} 153$ dipole moments in the ground and excited states, $a$ is the Onsager radius of the probe molecule, $\alpha_{g}$ and $\alpha_{\mathrm{e}}$ are the ground and excited state probe polarizabilities, $J$ and $J_{\mathrm{S}}$ are the probe and solvent ionization potentials and $h$ and $c$ have the usual meanings. These calculations were made assuming the same parameter values for C153 in the Franck-Condon $\left(S_{0}^{F C}, S_{1}^{F C}\right)$ and in the relaxed $\left(S_{0}^{r e l}, S_{1}^{r e l}\right)$ states, thus assuming the same dipole moment $\mu_{g}=6.6 \mathrm{D}$ [17] in $S_{0}^{F C}$ and $S_{0}^{r e l}, \mu_{e}=9.7 \mathrm{D}$ in $S_{1}^{F C}$ and $S_{1}^{r e l}$, and the same $\alpha_{g}$ in both $S_{0}$ states and $\alpha_{e}$ in both $S_{1}$ states [2]. EtOH ionization potential $J_{\mathrm{S}}=11.05 \mathrm{eV}$ was determined using AM1 hamiltonian with the MOPAC suite, EtOH $n(T)$ and $\varepsilon(\mathrm{T})$ were determined from the data given in [9]. For the other $\mathrm{C} 153$ and solvents parameters see $[1,2]$. The shifts in absorption of $\mathrm{C} 153$ in all solvents were predicted at subsequent temperatures. The values of $\Delta v_{\text {Abs }}$ were compared with experimental $\nu_{\mathrm{p}}(\mathrm{T})$ in the following way: for a selected solvent the absorption spectrum peak value $\nu_{\mathrm{p}}(293 \mathrm{~K})$ was added to the absolute value of the shift $\Delta v_{\mathrm{Abs}}$ (293 K). In this way a pseudo "gas-phase" $v_{p}^{g^{\prime \prime}}$ position of the spectrum was obtained. Next, from $v_{p}^{g^{\prime \prime}}$ the absolute values of $\Delta v_{\mathrm{Abs}}(\mathrm{T})$ were subtracted at subsequent temperatures different from $293 \mathrm{~K}$. The results of such a procedure are shown in Fig. 3 as solid lines. It led to the following $v_{p}^{g^{\prime \prime}}$ values: 26,060 $\mathrm{cm}^{-1}(\mathrm{ClH}), 26170(\mathrm{ClP}), 26120(\mathrm{PPN})$, $25840(\mathrm{EtOH})$ and 24920 (TFEtOH). As expected, $v_{p}^{g^{\prime \prime}}$ values are not the same as they were determined assuming that all solvents interacted exclusively nonspecifically which is obviously incorrect. But, they indicate which solvents most probably interact specifically with $\mathrm{C} 153$ in the ground state and these solvents are $\mathrm{EtOH}$ and TFEtOH. It is especially important that $v_{p}^{g^{\prime \prime}}$ in PPN fall into the same range 
as found for 1-chloroalkanes, and that the $v_{p}(\mathrm{~T})$ dependence slope in PPN corresponds exactly to the $\Delta v_{\text {Abs }}$ (T) dependence slope, see Fig. 3. Both these observations indicate that PPN interacts only nonspecifically with $\mathrm{C} 153$ in $S_{0}^{\text {rel }}$, or that the H-bond formed by PPN with $\mathrm{C} 153$ do not change in energy after $\mathrm{C} 153$ excitation to $S_{1}^{F C}$, contrary to what was deduced from the emissive results for $\mathrm{C} 153 S_{1}^{\text {rel }}$ in the same solvent [2]. On the other hand, a comparison of $v_{p}^{g^{\prime \prime}}$ values obtained in 1-chloroalkanes and PPN with those found in alcohols indicates that in EtOH a slight additional specific stabilisation take place already in $S_{1}^{F C}$, while in TFEtOH the specific interaction with $\mathrm{C} 153$ in $S_{1}^{F C}$ is significantly higher in energy than in $S_{0}^{r e l}$. No temperature dependence of this additional specific stabilisation is observed in $\mathrm{EtOH}$, while in TFEtOH its energy increases with decreasing temperature. This last result is in contrast to that found for $S_{1}^{r e l}$ [2]. It means that the Stokes shift in this solvent should decrease with decreasing temperature and indeed it is observed. Figure 7 shows the temperature dependence of the experimental Stokes shifts, $\Delta v_{\mathrm{S}}$, for $\mathrm{C} 153$ in all solvents.

The independence of $\Delta v_{S}$ of temperature in 1-chloroalkanes is unexpected as the emitting $S_{1}^{\text {rel }}$ state is more strongly stabilized than $S_{1}^{F C}$ due to the reorientational solvation of the solvent taking place when $\mathrm{C} 153$ is relaxed. The energy of this additional stabilizing interaction should increase with decreasing temperature, which in turn should lead to an increase in $\Delta v_{\mathrm{S}}$. However, the Onsager model (Eqs. 1 and 2 in [2]) reveal that the theoretical Stokes shift in $\mathrm{CIP}$ and $\mathrm{ClH}$ changes only by $90 \mathrm{~cm}^{-1}$ within the $120 \mathrm{~K}$ temperature interval studied in this work - a value in the range of the error of the points in Fig. 7 for $\mathrm{C} 153$ in 1-chloroalkanes. In PPN, EtOH and TFEtOH even a smaller $\Delta v_{\mathrm{S}}$ change is expected from the Onsager model. In contrast, clear $\Delta v_{\mathrm{S}}(\mathrm{T})$ dependencies in these solvents are observed. In TFEtOH $\Delta v_{\mathrm{S}}(\mathrm{T})$ dependence reflects the above-described effects. In PPN and EtOH the increase in $\Delta v_{\mathrm{S}}$ must be related to an additional stabilization of C153 in $S_{1}^{r e l}$ state, rising in energy with decreasing temperature. This

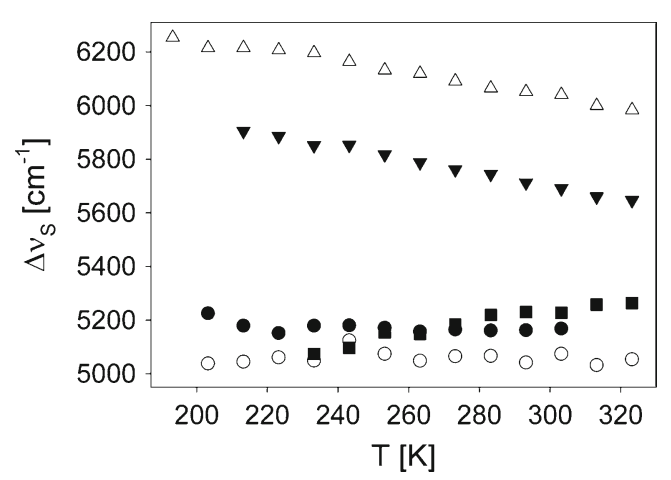

Fig. 7 Stokes shift vs temperature for C153 in: ClP (filled circles), $\mathrm{ClH}$ (empty circles), PPN (filled triangles), EtOH (empty triangles) and TFEtOH (filled squares) conclusion is in accordance with the results shown in [2]. The difference between the expected positions of the $\mathrm{C} 153$ emission spectra in methanol, PPN and TFEtOH and the experimental ones have been found to show temperature dependencies with the same slope signs as observed in Fig. 7 for $\Delta v_{\mathrm{S}}$ in the same two solvents. The analysis of the results given in [2] and in this work is based on the continuum solvent Onsager model, thus their reliability can be questioned by those who believe that this model fails in solvation description. Additionally, we have found the absorption spectrum full width at half of the maximum (fwhm) to be higher than that of the emission spectrum in all solvents, at each temperature. According to [18] such an observation can be a manifestation of a nonlinear dependence of the local solute potential on the solute dipole moment. This observation would however need a much deeper study, as absorption and emission spectra fwhm are strongly dependent on the frequency of the most active vibrational mode in a selected type of transition. Our results, shown later, and these presented in [15] reveal this frequency to be higher in the absorbing ground state, $S_{0}^{r e l}$, than in the emitting $S_{1}^{r e l}$. Nevertheless, the experimental $\Delta v_{\mathrm{S}}(\mathrm{T})$ dependence obtained for $\mathrm{C} 153$ in PPN compared to that found in 1-chloroalkanes gives a very strong argument for the presence of specific interactions between $\mathrm{C} 153$ in $S_{1}^{\text {rel }}$ and PPN molecules. Thus, the results show that $\mathrm{C} 153$ can act as a hydrogen donor. The presence of this type of H-bonding, however, does not influence $\mathrm{C} 153 \tau_{\mathrm{F}}(\mathrm{T})$ dependence, as can be deduced from results presented in Fig. 5. These results show that the protic character of the solvent has a significant impact on the $\tau_{\mathrm{F}}(\mathrm{T})$ dependence, when compared to nonprotic solvents (ClP as well [7]). Overall, the shortest lifetime is observed in the most protic TFEtOH, longer in $\mathrm{EtOH}, \mathrm{ClH}$, and the longest in PPN. In alcohols a slight modulation of the linear $\tau_{\mathrm{F}}(\mathrm{T})$ dependence can be observed, while in $\mathrm{ClH}$ and PPN the values of $\tau_{\mathrm{F}}$ changes with temperature in a similar way as in ClP, that is the slope of $\tau_{\mathrm{F}}(\mathrm{T})$ changes in sign at a temperature in the range $260 \div 280 \mathrm{~K}$. In both protic solvents a small modulation of $\tau_{\mathrm{F}}(\mathrm{T})$ takes place in the same temperature range in which the change in sign of the $\tau_{\mathrm{F}}(\mathrm{T})$ dependence occurs in $\mathrm{ClH}$ and PPN. Thus, we can assume that H-bonding with protic solvents dilutes $\mathrm{C} 153 \tau_{\mathrm{F}}(\mathrm{T})$ dependence resulting from pure intramolecular deactivation observed in $\mathrm{ClH}$ and PPN. Non-negligible is also the retarded solvation, which however as shown for $\mathrm{C} 153$ in EtOH is expected to lead to an increase in $\tau_{\mathrm{F}}$. The $\left.\tau_{\mathrm{F}} \mathrm{T}\right)$ dependence in neat solvents cover a narrow range of $\tau_{\mathrm{F}}$ values as can be seen in Fig. 6. Such subtle changes in $\tau_{\mathrm{F}}$ are also a manifestation of the narrow ranges in which $\phi_{\mathrm{F}}$ changes in all solvents. Together these quantities gives the radiative, $k_{\mathrm{F}}$, and non-radiative, $k_{\mathrm{nr}}$, rates. Figure 8 show their temperature dependencies for all four solvents. 

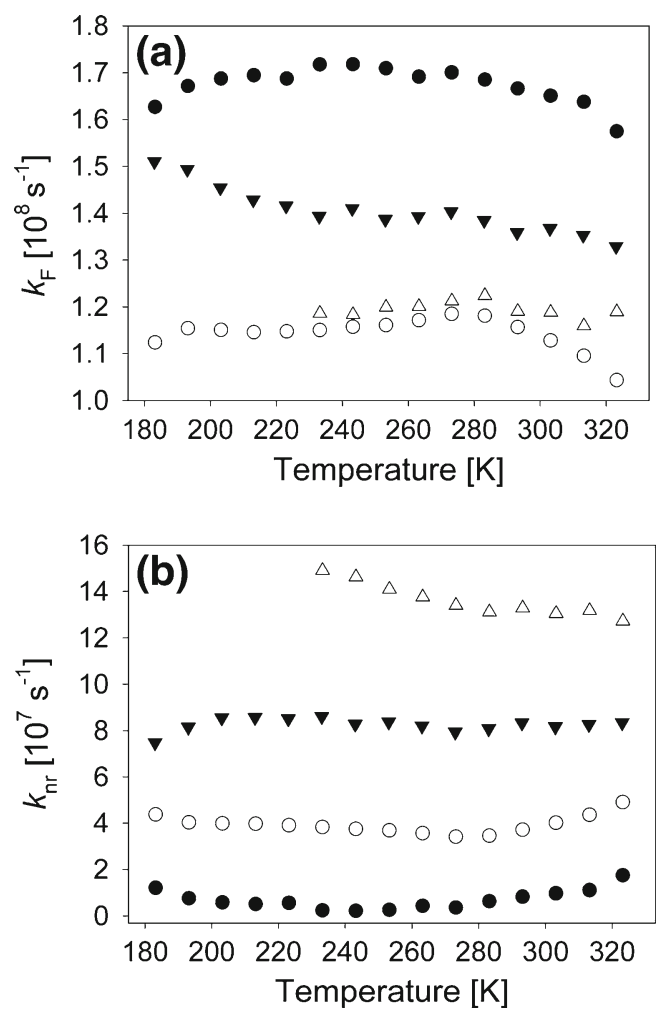

Fig. 8 Temperature dependencies of the radiative, (a) $k_{\mathrm{F}}$, and nonradiative, (b) $k_{\mathrm{nr}}$, rates in $\mathrm{ClH}$ (filled circles), PPN (empty circles), $\mathrm{EtOH}$ (filled triangles) and TFEtOH (empty triangles)

Non-radiative deactivation in $\mathrm{C} 153$ is known to be governed by internal conversion. According to our knowledge no ISC for this molecule has ever been observed. Relative $k_{\mathrm{nr}}$ values at the same temperatures in all solvents indicate that the energy-gap law partly controls non-radiative deactivation. The order in which $k_{\mathrm{nr}}$ values increase at a selected temperature corresponds well to the energy of emission in the solvents, thus to the emission position. However, the energy-gap law predicts an exponential decay of $k_{\mathrm{nr}}$ with temperature rising [19,20]. The temperature range in which measurements were made does not correspond to the tail of decaying $k_{\mathrm{nr}}(\mathrm{T})$ dependence, see Fig. 8 in [7]. In EtOH the non-exponentiality is evident in the low temperature range. However, in this solvent the retardation of the solvent relaxation is the source of the low-temperature $k_{\mathrm{nr}}(\mathrm{T})$ dependence, as the emission spectrum is not shifted totally to the red, which in turn leads to a drop in $k_{\mathrm{nr}}$ at the lowest T. In $\mathrm{ClH}$ and PPN $k_{\mathrm{nr}}(\mathrm{T})$ slope sign changes at higher temperatures. Similarly to what was observed for C153 in ClP [7], this effect is in conflict with the energy-gap law. Radiative rate $k_{\mathrm{F}}(\mathrm{T})$ dependencies have also similar features in all solvents. Using them, the modulus squared emission transition dipole moments at subsequent temperatures were determined, as shown in Fig. 9. These values were found using the Birks equation [21]:

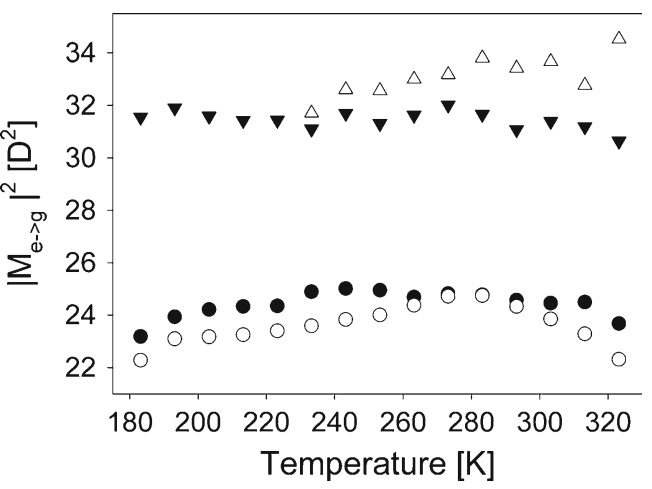

Fig. 9 Modulus squared of fluorescence $\left(\mathrm{M}_{\mathrm{e} \rightarrow \mathrm{g}}\right)$ transition dipole moments in $\mathrm{ClH}$ (filled circles), PPN (empty circles), EtOH (filled triangles) and TFEtOH (empty triangles)

$|M|_{e \rightarrow g}^{2}=\frac{3 h}{64 \pi^{4}} \frac{1}{n^{3}} k_{F} \widetilde{v}_{F}^{-3}$,

$\widetilde{v}_{F}^{-3}=\frac{\int I(v) v^{-3} d v}{\int I(v) d v}$

Now, we see that the $S_{1}^{r e l} \rightarrow S_{0}^{F C}$ radiative transition probability differs in protic solvents from that in nonprotic ones. Once again we stress that the results at lower temperatures in alcohols (especially in EtOH) are not reliable. Except for PPN, the T dependencies in the other solvents are not as well defined as for $\tau_{\mathrm{F}}$. It is connected to the noise in $\phi_{\mathrm{F}}$ and to the range of values in which $\tau_{\mathrm{F}}$ changes in a particular solvent. We would like to point out that $\tau_{\mathrm{F}}(\mathrm{T})$ results are blurred by a much smaller relative noise, comparing to $\phi_{\mathrm{F}}(\mathrm{T})$, which was determined indirectly from the results obtained by two different instruments. However, in $\mathrm{ClH}$ similarly as in PPN, we can see that $\mathrm{M}_{\mathrm{e} \rightarrow \mathrm{g}}$ starts to rise with $\mathrm{T}$ increasing from $183 \mathrm{~K}$ to end by falling at the highest temperature. It is also interesting to notice that except for the points at $323 \mathrm{~K}, \mathrm{M}_{\mathrm{e} \rightarrow \mathrm{g}}$ follows similar changes (increase/ decrease) in both protic solvents. It suggests that an additional modulation of $\mathrm{M}_{\mathrm{e} \rightarrow \mathrm{g}}$ may be present in both solvents, however it is out of reach of the resolution of our equipment. Changes in $\mathrm{M}_{\mathrm{e} \rightarrow \mathrm{g}}$ indicate changes in the equilibrium distance between $\mathrm{C} 153 S_{0}$ and $S_{1}$ potential energy surfaces. To check whether steady-state emission of $\mathrm{C} 153$ in PPN and $\mathrm{ClH}$ reflects such changes, the same model of emission spectrum was applied as in [7], described in [22,23]. According to this model, the emission spectrum of the molecule in a homogeneous noninteracting solvent can be defined as (MKS):

$I(v)=\sum_{n_{1}} \sum_{n_{2}} \ldots \sum_{n_{N}} I_{n_{1} n_{2} \ldots n_{N}}(v)$ 


$$
\begin{aligned}
& I_{n_{1} n_{2} \ldots n_{\mathrm{N}}}(v)=\left(\frac{E_{00}-\sum_{\mathrm{j}=1}^{\mathrm{N}} n_{\mathrm{j}} h v_{\mathrm{j}}}{E_{00}}\right)^{3} \prod_{\mathrm{j}=1}^{\mathrm{N}}\left(\frac{\xi_{\mathrm{j}}^{n_{\mathrm{j}}}}{n_{\mathrm{j}} !}\right) \\
& \exp \left[-4(\ln (2))\left(\frac{h v-E_{00}+\sum_{\mathrm{j}=1}^{\mathrm{N}} n_{\mathrm{j}} h v_{\mathrm{j}}}{\Delta v_{1 / 2}}\right)^{2}\right] \\
& \xi_{j}=1 / 2\left(\Delta_{j}\right)^{2}
\end{aligned}
$$

Emission intensity is given in units of the number of quanta emitted per energy interval [23]. The number of modes is truncated to $\mathrm{N}$ most active accepting vibrational modes in the ground state. $E_{00}$ is the energy gap between the lowest levels of the fitted vibronic modes of $S_{0}$ and $S_{1}$ states. The parameter $n_{\mathrm{j}}$ is the vibronic number of the $\mathrm{j}$-th accepting mode. The summations in Eqs. $(2,3)$ has been limited to $\mathrm{N}=1, n_{1}=6$. The symbol $v_{\mathrm{j}}$ is the $\mathrm{j}$-th accepting mode frequency. As the modes number was set to unity, we use below a substitution $\mathrm{j}=\mathrm{A}$, while $\nu$ is the frequency at which the emission spectrum is measured, $\Delta \bar{v}_{1 / 2}$ is the full width at half-maximum of the Gaussian inhomogeneous broadening, and $\bar{\xi}_{\mathrm{A}}$ is the HuangRhys factor related to the equilibrium bond distance difference, $\bar{\Delta}_{\mathrm{A}}$, between the excited and ground states, that is the parameter we are interested in. The function given in Eq. (2) served as a fitting model of the emission spectra at subsequent temperatures. $E_{00}, \bar{v}_{\mathrm{A}}, \bar{\xi}_{\mathrm{A}}, \Delta \bar{v}_{1 / 2}$ and a scaling $a_{0}$ factor (by which $I(\nu)$ was multiplied) were parameters of the fit. The overlines in the symbols indicates $\bar{v}_{\mathrm{A}}$ is in fact an average mode. The quality of the fit was estimated using the $\chi^{2}$ value and the errors of the fitted parameters values.

Similar results were obtained in both solvents, except for $E_{00}$ which included a solvent induced shift, thus it was higher in $\mathrm{ClH}$ than in PPN (Fig. 10). There is a correlation between $\bar{v}_{\mathrm{A}}(\mathrm{T}), \Delta \bar{v}_{1 / 2}$ and $\bar{\xi}_{\mathrm{A}}(\mathrm{T})$ dependencies, with significant changes in the same temperature range in which $\tau_{\mathrm{F}}(\mathrm{T})$ is observed to change. At this stage it is not possible to deduce how much these correlations affect the results of the fit, thus, to what extent $\bar{v}_{\mathrm{A}}(\mathrm{T}), \Delta \bar{v}_{1 / 2}$ and $\bar{\xi}_{\mathrm{A}}(\mathrm{T})$ dependencies reflects a true physical effect. These values are averages reflecting changes in different accepting modes properties [22,23]. The similarity between $\bar{v}_{\mathrm{A}}, \Delta \bar{v}_{1 / 2}$ and the corresponding quantities in [13] shows that the $1,150 \mathrm{~cm}^{-1}$ (in Fig. $10 \sim 1,250 \mathrm{~cm}^{-1}$ ) is the dominant mode in the emission process. However, $\bar{\xi}_{\mathrm{A}}(\mathrm{T})$ changes are hard to explain on the basis of a single mode deactivation, as they would indicate a rise and then a fall in equilibrium distance with decreasing temperature for C153 in
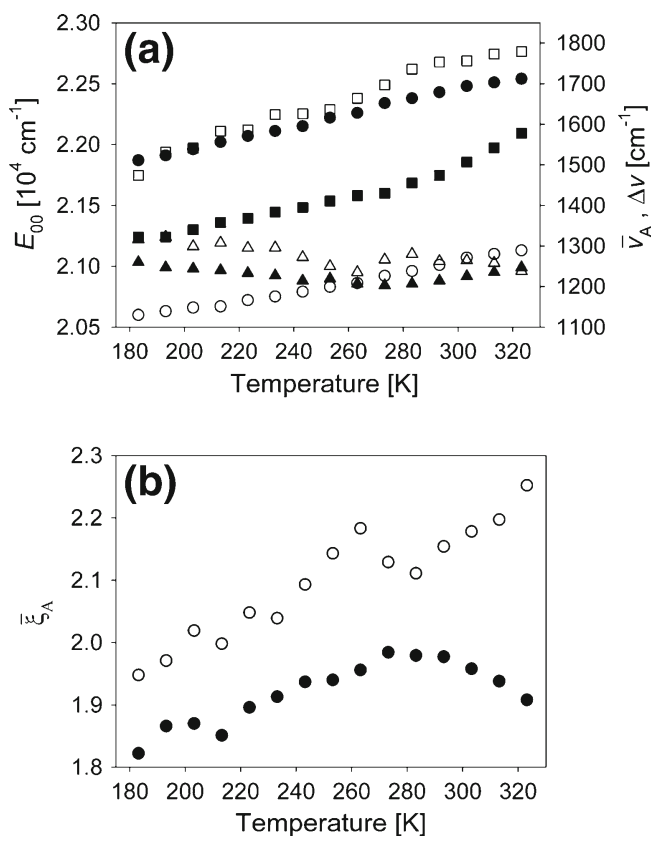

Fig. 10 Temperature dependence of: (a) $E_{00}, \mathrm{~S}_{1}-\mathrm{S}_{0}$ energy gap for $\mathrm{C} 153$ in $\mathrm{ClH}$ (filled circles) and PPN (empty circles), $\Delta \bar{v}_{1 / 2}$-full width at half-maximum in $\mathrm{ClH}$ (filled squares) and PPN (empty squares), $\bar{v}_{\mathrm{A}}$-in $\mathrm{ClH}$ (filled triangles) and PPN (empty triangles); (b) $\bar{\xi}_{\mathrm{A}}-$ Huang-Rhys vibrational coupling factor in $\mathrm{ClH}$ (filled circles) and PPN (empty circles) as obtained from fit of $\mathrm{C} 153$ emission spectra in both solvents to the model given in Eqs. (4-6)

ClH. In PPN an additional preliminary fall in this distance would be expected. However, assuming also that other accepting modes, as the $810 \mathrm{~cm}^{-1}$ and $360 \mathrm{~cm}^{-1}$ discussed in [7,13], are active in the fluorescence transition a quite simple explanation of the temperature dependence of $\mathrm{C} 153$ radiative deactivation can be proposed.

Both steady-state absorption (Fig. 2) and fluorescence results (Fig. 10) indicate that a change in the equilibrium distance $\bar{\Delta}_{\mathrm{A}}$ occurs on decreasing temperature. According to the Born-Oppenheimer approximation the transition dipole moment between the excited $\left(\psi^{\prime \prime}\right)$ and ground $\left(\psi^{\prime}\right)$ states is proportional to [24]:

$M_{e \rightarrow g}=\left\langle\psi^{\prime}|\widehat{\mu}| \psi^{\prime \prime}\right\rangle \propto P_{e l}(\bar{R}) \cdot \int \psi^{\prime} *_{v i b} \psi_{v i b}^{\prime \prime} d R$,

where $P_{e l}(\bar{R})$ is the purely electronic transition dipole moment, dependent on the $R$-centroid for the transition. The integral represents the overlap of the vibrational wavefunctions in both electronic states. Assuming the harmonicity of the oscillators representing molecular vibrations and no changes in the frequency of a selected oscillator between electronic states involved in the transition, one can quickly check that for a selected vibration mode (frequency) and two selected members of this mode progression in $S_{0}$ and $S_{1}$ states the integral in Eq. $7 M_{v i b}=\int \psi^{\prime} *_{v i b} \psi_{v i b}^{\prime \prime} d R$ can oscillate with $\bar{\Delta}_{\mathrm{A}}$ changed in the way shown in Fig. 11. 


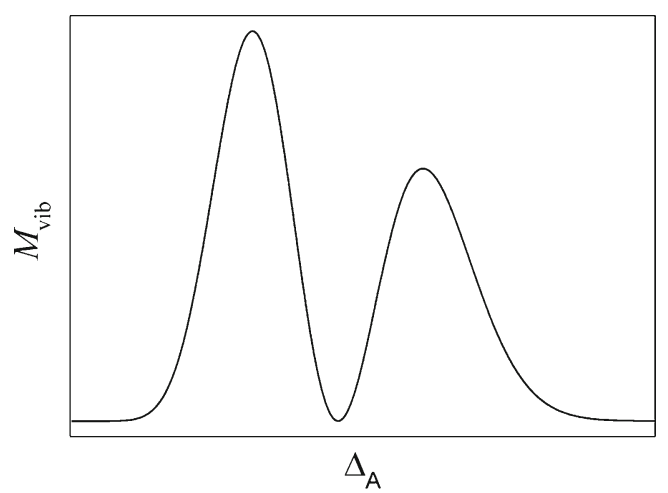

Fig. 11 Oscillation with change in equilibrium distance of the FranckCondon integral of two $S_{0}$ and $S_{1}$ harmonic oscillator wavefunctions corresponding to the same vibrational mode and two different members of its progression

Thus, changes in $\bar{\Delta}_{\mathrm{A}}$ can be the source of similar slight modulation in $\mathrm{M}_{\mathrm{e} \rightarrow \mathrm{g}}$ for $\mathrm{C} 153$ in $\mathrm{EtOH}$ and TFEtOH, or in $\mathrm{PPN}$ and $\mathrm{ClH}$. Both cases correspond to two different vibrational modes, in alcohols a high frequency one while in PPN and $\mathrm{ClH}$ a low frequency one. The modulation in $\mathrm{M}_{\mathrm{e} \rightarrow \mathrm{g}}(\mathrm{T})$ is reflected in the modulations in $\tau_{\mathrm{F}}(\mathrm{T})$. Changes in the equilibrium distance must result from changes in the solute-solvent interaction energies.

C153 clearly interacts specifically with protic solvents. Such a conclusion follows from the differences in $\mathrm{M}_{\mathrm{e} \rightarrow \mathrm{g}}(\mathrm{T})$ (Fig. 8) for aprotic and protic solvents and from the values of $\tau_{\mathrm{F}}(\mathrm{T})$ obtained for aqueous $\mathrm{ClH}$ and PPN compared to the ones obtained for the same solvents when dried properly (Fig. 6). It is also supported by steady-state absorption results. In aqueous $\mathrm{ClH}$ and PPN the decrease in $\mathrm{T}$ changes the equilibrium between free $\mathrm{C} 153$ molecules concentration and $\mathrm{C} 153+\mathrm{H}_{2} \mathrm{O}$ complexes in favor of the former ones. This is related to the fact water forms clusters of the size increasing with decreasing $\mathrm{T}[12,25]$, thus reducing the concentration of water molecules accessible for $\mathrm{C} 153$ to form complexes. The difference in $\mathrm{M}_{\mathrm{e} \rightarrow \mathrm{g}}$ and $k_{\mathrm{nr}}$ must be small for $\mathrm{C} 153$ and its complex with water and we observe an average result as the decay time. This is the case totally different from that of 4-AP which is efficiently quenched by water $\mathrm{H}$-bonding, responsible for the decay times of free 4-AP and 4-AP-water complexes significantly different and separable through optimization routines.

Comparison of $\mathrm{C} 153 \mathrm{M}_{\mathrm{e} \rightarrow \mathrm{g}}$ values in protic and aprotic solvents indicates that $\mathrm{C} 153 \mathrm{H}$-bonding of molecules of protic solvents lead to a change in the equilibrium distance $\Delta_{A}$ or/and to a slight change in $S_{0}$ and $S_{1}$ potential energy surfaces shapes. Simultaneously, the steady-state absorption results show that the same interaction in the same solvents leads to an additional stabilization of the $S_{1}^{F C}$ state with respect to $S_{0}$, when compared to what is observed in aprotic solvents. In $S_{1}^{r e l}$ state H-bonds formed by C153 with PPN change in energy which leads to an additional stabilization of the emitting state. We can assume that the same process takes place in EtOH. Such a conclusion follows from Stokes shifts values $\left(\Delta v_{\mathrm{S}}\right.$, Fig. 7). On the other hand, the absorption position temperature dependencies (Fig. 3) and $\Delta v_{\mathrm{S}}(\mathrm{T})$ show that in protic TFEtOH the energy of H-bonds formed with C153 decreases with decreasing temperature in the probe $S_{1}^{\text {rel }}$ state, in contrast to what is observed for $S_{1}^{F C}$. In hydrogen accepting PPN the H-bonds become stronger with decreasing T for $\mathrm{C} 153$ in $S_{1}^{r e l}$ state. In EtOH an average result of both processes is observed.

\section{Conclusions}

Overall, we can conclude that the $S_{0}$ and $S_{1}$ potential energy surfaces undergo two displacements upon changing the solvent and temperature. Horizontal displacement along the equilibrium distance axis induce changes in $\mathrm{M}_{\mathrm{e} \rightarrow \mathrm{g}}, \tau_{\mathrm{F}}$ and $\phi_{\mathrm{F}}$. Vertical displacement in the energy scale changes the absorption and emission positions and $k_{\mathrm{NR}}$, which thus affects $\tau_{\mathrm{F}}$ and $\phi_{\mathrm{F}}$. Temperature changes imply changes in nonspecific interaction energy. Thus, the induced part of the total dipole moment of the $\mathrm{C} 153$ molecule is modified as well. This in turn involves changes in $\mathrm{H}$-bonds energies formed by the probe with TFEtOH, EtOH and PPN in $\mathrm{S}_{0}$ and $\mathrm{S}_{1}$, of Franck-Condon and relaxed character. At this stage a direct indication of $\mathrm{C} 153$ sites/atoms responsible for the experimental effects observed is out of reach. However, on the basis of the steady-state results we can safely conclude that the reorientational relaxation of the solvent accompanying the $S_{1}^{F C} \rightarrow S_{1}^{\text {rel }}$ transition induces a weakening of the H-bonds formed by $\mathrm{C} 153$ with protic solvents and a rise in energy of the H-bonds formed with hydrogen accepting solvents. This effects is amplified by a decrease in $\mathrm{T}$ and it can be understood as a result of a competition between specific and nonspecific interactions in TFEtOH, and as a result of cooperation between them in PPN. One should keep in mind that in both $S_{1}$ states the H-bond energy is higher than in $S_{0}$ in protic solvents, while in PPN the Hbond energy in $S_{1}^{F C}$ is the same as in $S_{0}$.

Acknowledgment This study was performed under financial support of the Polish Ministry of Science and Higher Education (project N N202 091339). Time-resolved fluorescence and steady-state studies were performed at the Center for Ultrafast Laser Spectroscopy of A. Mickiewicz University in Poznań. We thank Wojciech Jarmużek for technical support.

Open Access This article is distributed under the terms of the Creative Commons Attribution License which permits any use, distribution, and reproduction in any medium, provided the original author(s) and the source are credited. 


\section{References}

1. Dobek K (2008) Temperature influence on the energy of nonspecific and specific interactions taking place between 4-aminophthalimide (4-AP) and homogeneous solvents. Photochem Photobiol Sci 7 (3):361-370

2. Tomczak J, Dobek K (2009) Coumarin 153 emission thermochromism studied in non-specifically and specifically interacting solvents. J Lumin 129(8):884-891

3. Hagan T, Pilloud D, Suppan P (1987) Thermochromic shifts of some molecular and exciplex fluorescence spectra. Chem Phys Lett 139(6):499-502

4. Ghoneim N, Rocher Y, Suppan P (1988) Solvathochromic and thermochromic effects in low-temperature rigid matrices. Faraday Discuss Chem Soc 86:295-308

5. Suppan P (1990) Solvatochromic shifts: the influence of the medium on the energy of electronic states. J Photochem Photobiol A 50(3):293-330

6. Noukakis D, Suppan P (1991) Photophysics of aminophthalimides in solution I. Steady-state spectroscopy. J Lumin 47(6):285-295

7. Dobek K (2011) The influence of temperature on coumarin 153 fluorescence kinetics. J Fluoresc 21(4):1547-1557

8. Kamlet MJ, Taft RW (1976) The solvatochromic comparison method. I. The $\beta$-scale of solvent hydrogen-bond acceptor (HBA) basicities. J Am Chem Soc 98(2):377-383

9. Kamlet MJ, Taft RW (1976) The solvatochromic comparison method. 2. The $\alpha$-scale of solvent hydrogen-bond donor (HBD) acidities. J Am Chem Soc 98(10):2886-2894

10. Marcus Y (1998) The properties of solvents. Wiley, Baffins Lane, Chichester

11. Karolczak J, Komar D, Kubicki J, Wróżowa T, Dobek K, Ciesielska B, Maciejewski A (2001) The measurements of picosecond fluorescence lifetimes with high accuracy and subpicosecond precision. Chem Phys Lett 344(1-2):154-164

12. Dobek K, Karolczak J, Komar D (2012) Temperature influence on 4-aminophthalimide emission in 1-chloroalkanes plus water mixtures. J Phys Chem A 116(25):6655-6663
13. Mühlpfordt A, Schanz R, Ernsting NP, Farztdinov V, Grimme S (1999) Coumarin 153 in the gas phase: optical spectra and quantum chemical calculations. Phys Chem Chem Phys 1(7):32093218

14. Pryor BA, Palmer PM, Chen Y, Topp MR (1999) Identification of dual conformers of coumarin 153 under jet-cooled conditions. Chem Phys Lett 299(6):536-544

15. Horng ML, Gardecki JA, Papazyan A, Maroncelli M (1995) Subpicosecond measurements of polar solvation dynamics: coumarin 153 revisited. J Phys Chem 99(48):17311-17337

16. McRae EG (1957) Theory of solvent effects on molecular electronic spectra. Frequency shifts. J Phys Chem 61(5):562-572

17. Moylan CR (1994) Molecular hyperpolarizabilities of coumarin dyes. J Phys Chem 98(51):13513-13516

18. Matyushov DV, Ladanyi BM (1997) Nonlinear effects in dipole solvation. II. Optical spectra and electron transfer activation. J Chem Phys 107(5):1375-1387

19. Englman R, Jortner J (1970) The energy gap law for radiationless transitions in large molecules. Mol Phys 18(2):145-164

20. Freed KF, Jortner J (1970) Multiphonon processes in the nonradiative decay of large molecules. J Chem Phys 52(12):62726292

21. Birks JB (1970) Photophysics of aromatic molecules. Wiley, New York

22. Caspar JV, Westmoreland TD, Allen GH, Bradley PG, Meyer TJ, Woodruff WH (1984) Molecular and electronic structure in the metal-to-ligand charge-transfer excited states of d6 transitionmetal complexes in solution. J Am Chem Soc 106(12):34923500

23. Kober EM, Caspar JV, Lumpkin RS, Meyer T (1986) Application of the energy gap law to excited-state decay of osmium(II)-polypyridine complexes: calculation of relative nonradiative decay rates from emission spectral profiles. J Phys Chem 90(16):37223734

24. Levine IN (1974) Molecular spectroscopy. Wiley, New York

25. Gelman-Constantin J, Carignano MA, Szleifer I, Marceca EJ, Corti HR (2010) Structural transitions and dipole moment of water clusters $\left(\mathrm{H}_{2} \mathrm{O}\right)_{\mathrm{n}=4-100}$. J Chem Phys 133(2):024506 